\title{
Investigation of the Efficiency of Moment Method Solution Based on Expansion Functions Defined in the Infinite Domain for Electrostatic Problems
}

\author{
T. Teper, H. Matzner*, P. Malits \\ Department of Communication Engineering, Faculty of Engineering, HIT - Holon Institute of Technology, Golomb 52, Holon 58102, Israel
}

\begin{abstract}
The efficiency of the Moment Method (MM) when the expansion functions are defined in the infinite domain is checked. It is shown that efficient solution is obtained when the expansion functions obey the known physical behaviour of the fields. The age-old problem of the thin, charged disk is solved by the MM for which an electric field component is expanded outside the body. This solution is compared to the known analytic solution and to the MM solution for which the surface charge density is expanded on the finite disk. An excellent agreement between the analytical solution and the MM solution based on expansion functions defined in the infinite domain was achieved.
\end{abstract}

Keywords Moment Method, Entire Domain, Infinite Domain

\section{Introduction}

Moment Method (MM) is used frequently in solving electromagnetic problems[1]. Different expansion (basis) functions and test functions were checked during the last years in order to get more accurate and fast converging solutions. The inclusion of the edge behaviour of the fields in the expansion functions in order to get more accurate and fast converging solutions is addressed in many papers. For example[2] deals with the characterization of an infinite array microstrip reflectarray elements, and it is said that a complete set of trigonometric functions which do not enforce the correct edge conditions shows a slow rate of convergence of the solution. $\operatorname{In}[3]$ there is a use of MM basis functions containing the $90 \mathrm{o}$ conducting edge singularity behaviour to efficiently solve an infinite array of stub-loaded rectangular waveguides. It was shown, for example, by[4] that while expansion functions exactly obey the known physical behaviour of the fields, the solution obtained is more accurate and fast converging, as compared to cases when the expansion functions do not obey the known physical behaviour of the field. Fuzzy basis functions which can approximate any function to an arbitrary degree of accuracy are applied in[5]. In this method the user's insight can be taken into account easily and systematically resulting in a better approximation. Recently large complex structures are solved by the MM by dividing the

* Corresponding author:

meitarit@netvision.net.il (H. Matzner)

Published online at http://journal.sapub.org/ajcam

Copyright (C) 2012 Scientific \& Academic Publishing. All Rights Reserved structure to sub-domains, where few expansion functions are needed for each sub-domain. Use of these basis functions leads to a significant reduction in the number of unknowns, and results in a substantial size reduction of the MM matrix[6,7].

In this paper we check the efficiency of an MM solution when the expansion functions are defined in the infinite domain. To do this we choose a simple electromagnetic problem (a toy model) for which the analytic solution is known, and compare our MM solution based on expansion functions defined in the infinite domain to the analytic solution and to an MM solution based on expansion functions defined in the finite domain. The relevant problem chosen is the isolated flat, infinitely thin, ideal conducting circular disc of radius $\mathrm{R}$, with a total charge $\mathrm{q}$ placed on it. We choose to expand the $\rho$ component of the electric field outside the disk, where the expansion functions obey the exact physical behaviour of the field near the edge of the disk and at infinity. This solution in compared to the MM solution where the expansion functions describe the surface charge density on the finite disk.

The structure of the paper is as follows: chapter II describes the formulation of the problem and includes the analytical solution and the MM solutions. Chapter III deals with the selection of the expansion functions, and numerical results are presented in chapter IV. Finally, conclusions are discussed in chapter V. Some relevant mathematical calculations are presented in the Appendix.

\section{Formulation}

\subsection{Geometry and Analytic Solution}


The thin, charged disk is shown in figure 1 . The radius of the disk is $\mathrm{R}$ and the total charge on it is $\mathrm{q}$

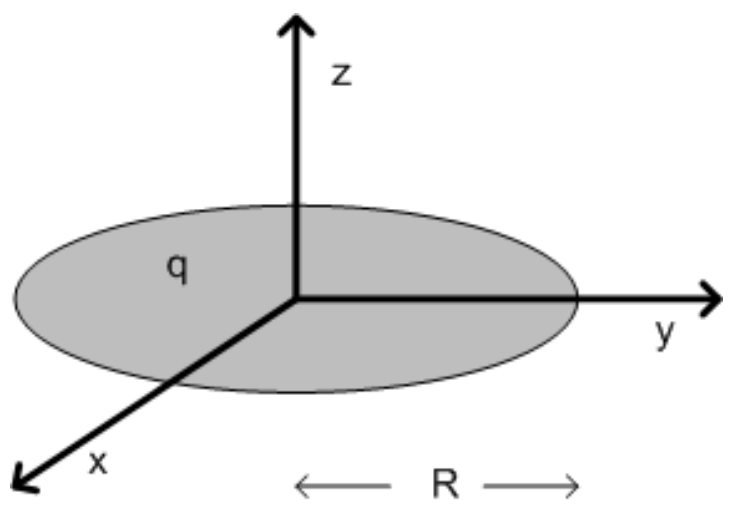

Figure 1. Isolated, infinitely thin, flat, circular conducting disk of radius $\mathrm{R}$ with a total charge $\mathrm{q}$ placed on it

The analytic solution for the surface charge distribution and $\mathrm{E}_{\rho}$ at $\mathrm{z}=0$ are given in the cylindrical system of coordinates $(\rho, \varphi, z)[8]$.

$$
\begin{gathered}
\sigma(\rho)=\frac{q}{2 \pi R} \frac{1}{\sqrt{R^{2}-\rho^{2}}}, 0 \leq \rho<R \\
E_{\rho}(\rho, z=0)=\left\{\begin{array}{lr}
0 & 0<\rho<R \\
\frac{1}{4 \pi \varepsilon_{0}} \cdot \frac{1}{\rho \sqrt{\rho^{2}-R^{2}}} & \rho>R
\end{array}\right.
\end{gathered}
$$

The analytic solution will be compared to the MM solutions based on expansion functions defined in the finite and in the infinite domains.

\subsection{The Fields Related to the Problem}

Laplace equation around the disk is specified by

$$
\nabla^{2} \phi=0
$$

where is the potential function. The solution is

$$
\phi(\rho, z)= \begin{cases}\phi_{1}(\rho, z), & z \leq 0 \\ \phi_{2}(\rho, z), & z \geq 0\end{cases}
$$

where

$$
\begin{gathered}
\phi_{1}(\rho, z)=\int_{0}^{\infty} A(k) J_{0}(k \rho) \exp (k z) d k \\
\phi_{2}(\rho, z)=\int_{0}^{\infty} A(k) J_{0}(k \rho) \exp (-k z) d k
\end{gathered}
$$

[8], and where $\mathrm{J}_{0}($.$) is the Bessel function of the first$ kind[9]. $\mathrm{A}(\mathrm{k})$ will be expressed by the MM expansion functions.. The electric field components are given by

$$
E_{\rho}=-\frac{\partial \phi}{\partial \rho} \quad, \quad E_{z}=-\frac{\partial \phi}{\partial z}
$$

\subsection{Equation Based on Expansion Functions Defined in the Infinite Domain}

Expanding E $\rho$ outside the disk, on the disk plane, by the MM set of expansion functions $\{\operatorname{ej}(\rho), j=1,2, . . N\}$ where $N$ is the number of expansion functions taken to the calculation, we have

$$
\begin{aligned}
E_{\rho}(\rho, z=0) & =-\left.\frac{\partial \phi_{1}}{\partial \rho}\right|_{z=0} \\
& =\left\{\begin{array}{cc}
\sum_{j=1}^{N} a_{j} e_{j}(\rho), \rho>R \\
0, & \rho \leq R
\end{array}\right. \\
& =\int_{0}^{\infty} A\left(k^{\prime}\right) J_{1}\left(k^{\prime} \rho\right) k^{\prime} d k^{\prime}
\end{aligned}
$$

Extracting $\mathrm{A}(\mathrm{k})$ in terms of the expansion functions by multiplying both sides of the former equation by $J_{1}(k \rho) \rho$ and integrate over $\rho$ from 0 to infinity

$$
\begin{aligned}
& \int_{0}^{\infty} \int_{0}^{\infty} A\left(k^{\prime}\right) J_{1}\left(k^{\prime} \rho\right) k^{\prime} J_{1}(k \rho) \rho d \rho d k^{\prime} \\
= & \int_{R}^{\infty} d \rho \sum_{j=1}^{N} a_{j} e_{j}(\rho) J_{1}(k \rho) \rho
\end{aligned}
$$

By using[10]

$$
\int_{0}^{\infty} \rho J_{1}(k \rho) J_{1}\left(k^{\prime} \rho\right) d \rho=\frac{1}{k} \delta\left(k-k^{\prime}\right)
$$

we obtain

$$
A(k)
$$

$$
=\int_{R}^{\infty} \sum_{j=1}^{N} a_{j} e_{j}(\rho) \rho J_{1}(k \rho) d \rho=\sum_{j=1}^{N} a_{j} \tilde{e}_{j}(k)
$$

where

$$
\tilde{e}_{j}(k)=\int_{R}^{\infty} e_{j}(\rho) J_{1}(k \rho) \rho d \rho
$$

The boundary condition for $\mathrm{Ez}$ is

$$
\left.\epsilon_{0}\left(E_{z 2}-E_{z 1}\right)\right|_{z=0}=\left\{\begin{array}{cc}
\sigma(\rho), & \rho \leq R \\
0, & \rho>R
\end{array}\right.
$$

Writing in terms of the potential, outside the disk

$$
-\left.\frac{\partial \phi_{1}}{\partial z}\right|_{z=0}-\left.\left(-\frac{\partial \phi_{2}}{\partial z}\right)\right|_{z=0}=0, \quad \rho>R
$$

Now expressing the potential in terms of $\mathrm{A}(\mathrm{k})$, the former equation is

$$
2 \int_{0}^{\infty} A(k) J_{0}(k \rho) k d k=0, \quad \rho>R
$$

Applying Galerkin method, we have ti $(\rho)=$ ei $(\rho), \mathrm{i}=$ $1,2, . . \mathrm{N}$, where $\operatorname{ti}(\rho)$ are the test functions. Multiply the former equation by $\operatorname{ti}(\rho) \rho$ for $i=1,2, . . N$, and integrate over $\rho$ from $\mathrm{R}$ to infinity, we get the equation

$$
\begin{aligned}
& \int_{R}^{\infty} t_{i}(\rho) \rho d \rho \int_{0}^{\infty} A(k) J_{0}(k \rho) k d k \\
= & \int_{0}^{\infty} A(k) \tilde{t}_{i}(k) k d k=0, \quad i=1,2 \ldots N
\end{aligned}
$$

where $\tilde{t}_{i}(k)$ is given by 


$$
\tilde{t}_{i}(k)=\int_{R}^{\infty} t_{i}(\rho) \rho J_{0}(k \rho) d \rho
$$

Finally we get the MM equation by expressing $\mathrm{A}(\mathrm{k})$ in terms of the expansion functions

$$
\int_{0}^{\infty} d k \sum_{j=1}^{N} a_{j} \tilde{e}_{j}(k) \tilde{t}_{i}(k) k=0 \quad, i=1,2 \ldots N(16)
$$

or in matrix notation

$$
B a=0
$$

where

$$
B_{i j}=\int_{0}^{\infty} \tilde{t}_{i}(k) \tilde{e}_{j}(k) k d k
$$

and where $\mathrm{a}=\left(\mathrm{a}_{1}, \mathrm{a}_{2}, \ldots\right)$ is the vector containing the un normalized MM expansion function coefficients. The final values of the coefficients are determined by taking into account the value of the total charge on the disk.

\subsection{Equation Based on Expansion Functions Defined in the Finite Domain}

In this case we expand the surface charge density

$$
\sigma(\rho)=\left\{\begin{array}{cc}
\sum_{j=1}^{N} b_{j} f_{j}(\rho) & \rho \leq R \\
0 & R \leq \rho \leq \infty
\end{array}\right.
$$

The boundary condition for $\mathrm{E}_{\mathrm{z}}$ is

$$
-\left.\frac{\partial \phi_{1}}{\partial z}\right|_{z=0}-\left.\left(-\frac{\partial \phi_{2}}{\partial z}\right)\right|_{z=0}=\frac{\sigma(\rho)}{\varepsilon_{0}}
$$

Expressing the surface charge density in terms of $A(k)$, we obtain

$$
\sigma(\rho)=2 \epsilon_{\mathrm{O}} \int_{\mathrm{O}}^{\infty} A\left(k^{\prime}\right) J_{\mathrm{O}}\left(k^{\prime} \rho\right) k^{\prime} d k^{\prime}
$$

Multiplying both sides of the former equation by $\mathrm{J}_{0}(\mathrm{k} \rho) \rho$ and integrate over $\rho$ from 0 to infinity, we have for $A(k)$

$$
2 \varepsilon_{0} A(k)=\sum_{j=1}^{N} b_{j} \tilde{f}_{j}(k)
$$

where

$$
\widetilde{f}_{j}(k)=\int_{0}^{R} f_{j}(\rho) J_{0}(k \rho) \rho d \rho
$$

Now, setting the boundary condition

$$
E_{\rho}=0 \quad 0<\rho \leq R, \quad z=0
$$

and expressing $\mathrm{E}_{\rho}$ on the disk in terms of the expansion functions

$$
\begin{aligned}
E_{\rho} & =\int_{0}^{\infty} A(k) J_{1}(k \rho) k d k \\
& =\int_{0}^{\infty} J_{1}(k \rho) k d k \sum_{j=1}^{N} \frac{b_{j}}{2 \varepsilon_{0}} \widetilde{f}_{j}(k)=0 \\
0 & <\rho \leq R
\end{aligned}
$$

Now we can obtain the MM equation: multiply both sides of the last equation by $\mathrm{f}_{\mathrm{i}}(\rho) \rho$ and integrate from 0 to $\mathrm{R}$

$$
\begin{aligned}
& \int_{0}^{R} f_{i}(\rho) \rho\left\{\int_{0}^{\infty} J_{1}(k \rho) k d k \sum_{j=1}^{N} b_{j} \widetilde{f}_{j}(k)\right\} d \rho \\
= & 0, \quad i=1,2, . N
\end{aligned}
$$

By changing order of integration and summation, we can write the former expression in a more compact form

$$
\widetilde{t}_{i}(k)=\int_{0}^{R} f_{i}(\rho) J_{1}(k \rho) \rho d \rho
$$

where

$$
\sum_{j=1}^{N} b_{j} \int_{0}^{\infty} \widetilde{f}_{j}(k) \widetilde{t}_{i}(k) k d k=0
$$

and in matrix notation

$$
B b=0, \quad B_{i j}=\int_{0}^{\infty} \widetilde{f}_{j}(k) \widetilde{t}_{i}(k) k d k
$$

where $b=\left(b_{1}, b_{2}, \ldots\right)$ is the vector containing the un-normalized MM expansion function coefficients. The final values of the coefficients are determined by taking into account the value of the total charge on the disk.

\subsection{Choosing the Expansion Functions in the Infinite Domain}

The expansion functions for $E_{\rho}(\rho, z=0)$ are chosen such to obey the physical behaviour of this field component near the edge of the disk and at infinity. The behaviour is given by $E_{\rho}(\rho, z=0)$

$$
\sim\left\{\begin{array}{c}
0 \quad \rho \leq R \\
\alpha_{1}(\rho-R)^{-1 / 2}+\alpha_{2}(\rho-R)^{1 / 2} \\
+\alpha_{3}(\rho-R)^{3 / 2}+\ldots, \\
\rho \rightarrow R, \quad \rho>R \\
\frac{\beta_{1}}{\rho^{2}}+\frac{\beta_{2}}{\rho^{4}}+\frac{\beta_{3}}{\rho^{6}}+\ldots, \quad \rho \rightarrow \infty
\end{array}\right.
$$

where $\alpha_{1}, \alpha_{2}, . ., \beta_{1}, \beta_{2}, .$. are constants. It can be shown that the following functions belonging to the two sets below for $\mathrm{E}_{\rho}(\rho, \mathrm{z}=0)$

$$
\begin{gathered}
\left\{\begin{array}{cc}
\frac{1}{\rho^{2(j-1) \sqrt{\rho^{4}-R^{4}}}}, \\
j=1,2,3, \ldots, & \rho>R
\end{array}\right\} \\
\left\{\begin{array}{cc}
\frac{\sqrt{\rho^{4}-R^{4}}}{\rho^{2 j}}, & \\
j=2,3, \ldots, & \rho>R
\end{array}\right\}
\end{gathered}
$$

contain all the needed powers near the edge of the disk and at infinity. Note that these functions do not include 'wrong' powers, that is, powers which do not belong to the correct edge powers for $\rho \rightarrow \mathrm{R}$ and/or for $\rho \rightarrow \infty$. In addition, these functions have analytical Fourier-Bessel transform, in terms of the generalized hypergeometric functions[11]. The analytic solution is not included in the expansion function sets. 


\subsection{Choosing the Expansion Functions in the Finite Domain}

The a-priory physical behaviour if the surface charge density known to us is

$$
\sim\left\{\begin{array}{c}
\sigma(\rho) \\
b_{1}(R-\rho)^{-1 / 2}+b_{2}(R-\rho)^{1 / 2} \\
+\alpha_{3}(R-\rho)^{3 / 2} \ldots, \\
\rho \rightarrow R, \quad \rho<R \\
c_{0}+c_{1} \rho^{2}+c_{2} \rho^{4}+\ldots, \quad \rho \rightarrow 0
\end{array}\right.
$$

where $\mathrm{b} 1, \mathrm{~b} 2, \ldots \mathrm{c} 0, \mathrm{c} 1, .$. are constants. It can be shown that the functions belonging to the set below for $\sigma(\rho)$

$$
\left\{\frac{T_{2(j-1)}(\rho)}{\sqrt{R^{4}-\rho^{4}}}, \quad j=1,2,3, \ldots, \quad \rho<R\right\} \text { (34) }
$$

contain all the required powers when $\rho \rightarrow 0$ and when $\rho \rightarrow \mathrm{R}$, where $\mathrm{Ti}($.$) are the Chebyshev polynomials[12].$ Note that these functions do not include 'wrong' powers, that is, powers which do not belong to the correct edge powers for $\rho \rightarrow \mathrm{R}$ and/or for $\rho \rightarrow 0$. In addition, these functions have analytical Fourier-Bessel transform in terms of the generalized hypergeometric functions. These expansion functions do not contain the analytic solution.

\section{Numerical Results}

3.1 MM Solution Based on Expansion Functions Defined in the Infinite Domain

The expansion functions taken for the calculation were

$$
\begin{aligned}
& e_{1}(\rho)=\frac{1}{\sqrt{\rho^{4}-R^{4}}} ; e_{2}(\rho)=\frac{1}{\rho^{2} \sqrt{\rho^{4}-R^{4}}} \\
& e_{3}(\rho)=\frac{\sqrt{\rho^{4}-R^{4}}}{\rho^{4}} ; e_{4}(\rho)=\frac{\sqrt{\rho^{4}-R^{4}}}{\rho^{6}}
\end{aligned}
$$

The MM matrix elements where analytically calculated by MATHEMATICA software[13]. The results for the expansion functions coefficients are shown in table 1.

The surface charge density is shown in fig. 2, where for simplicity we have set $\mathrm{R}=1$. It is seen that an excellent rate of convergence for the solution was achieved. The red line for 4 expansion functions overlaps the brown line for 2 expansion functions, the yellow line for 3 expansion functions, and the green line which presents the analytic solution.

A zoom on the area near the centre of the disk is shown in fig. 3

Table 1. MM Coefficients of the expansion functions defined in the infinite domain

\begin{tabular}{|c|c|c|c|c|}
\hline & 1 & 2 & 3 & 4 \\
\hline 1 & 0.21049 & & & \\
\hline 2 & 0.16277 & 0.06265 & & \\
\hline 3 & 0.14912 & 0.07595 & 0.01032 & \\
\hline 4 & 0.14275 & 0.08233 & 0.01644 & -0.00348 \\
\hline
\end{tabular}

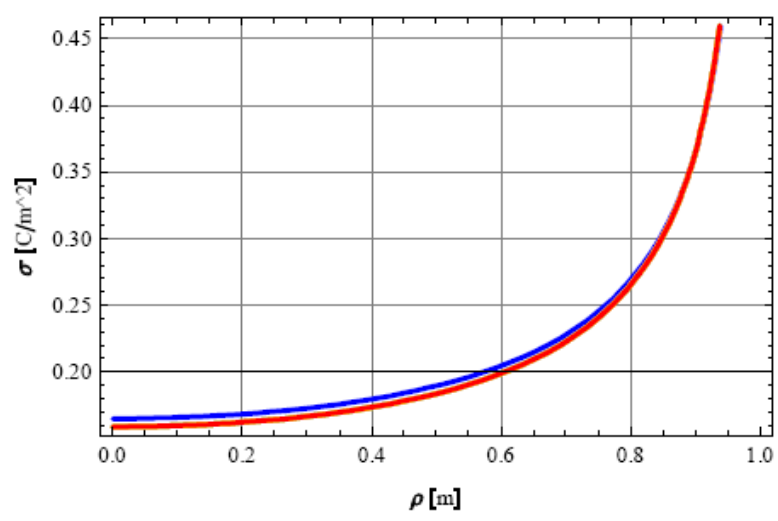

Figure 2. Surface charge distribution on the disk, based on expansion functions defined in the infinite domain. Green line is for the analytic solution, blue - for one expansion function, brown - for two expansion functions, yellow - for three expansion function, and red line is for four expansion functions

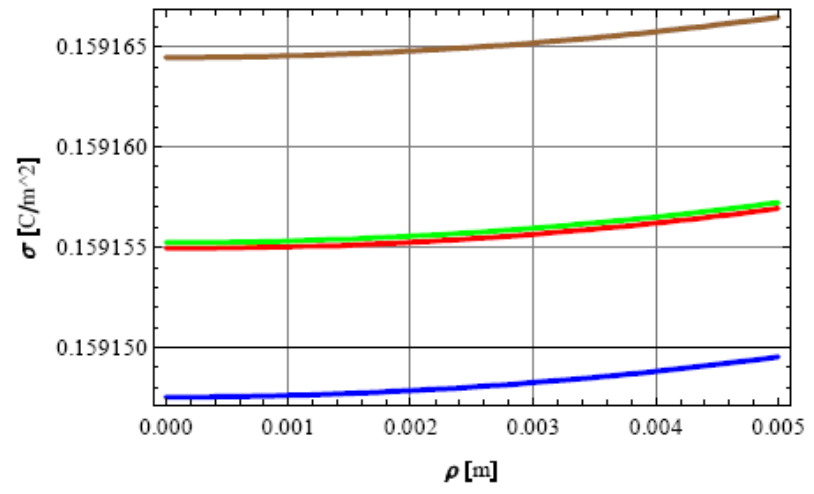

Figure 3. Surface charge distribution near the centre of the disk, based on expansion functions defined in the infinite domain.. The red line is for the analytic solution, brown - for two expansion functions, blue - for three expansion function, and green line is for four expansion functions

\subsection{Solution Based on Expansion Functions Defined in the Finite Domain}

The three expansion functions fi $(\rho), i=1,2,3$ for the surface charge density on the disk taken to the calculation were, respectively

$$
\frac{1}{\sqrt{R^{4}-\rho^{4}}} ; \frac{-1+2 \rho^{2}}{\sqrt{R^{4}-\rho^{4}}} ; \frac{1-8 \rho^{2}+8 \rho^{4}}{\sqrt{R^{4}-\rho^{4}}}
$$

The MM matrix elements where analytically calculated by MATHEMATICA softwar. The results for the expansion function coefficients are shown in table 2.

The surface charge density is shown in fig. 4, exhibits an excellent rate of convergence for the solution. The green line for 3 expansion functions overlaps the blue line for 2 expansion functions, and the red which presents the analytic solution.

A zoom on the area near the centre of the disk is shown in fig. 5.

Table 2. MM Coefficients of the expansion functions defined in the finite domain

\begin{tabular}{|c|c|c|c|}
\hline & 1 & 2 & 3 \\
\hline 1 & 0.202642 & & \\
\hline 2 & 0.194083 & 0.031324 & \\
\hline 3 & 0.193429 & 0.032805 & -0.002690 \\
\hline
\end{tabular}




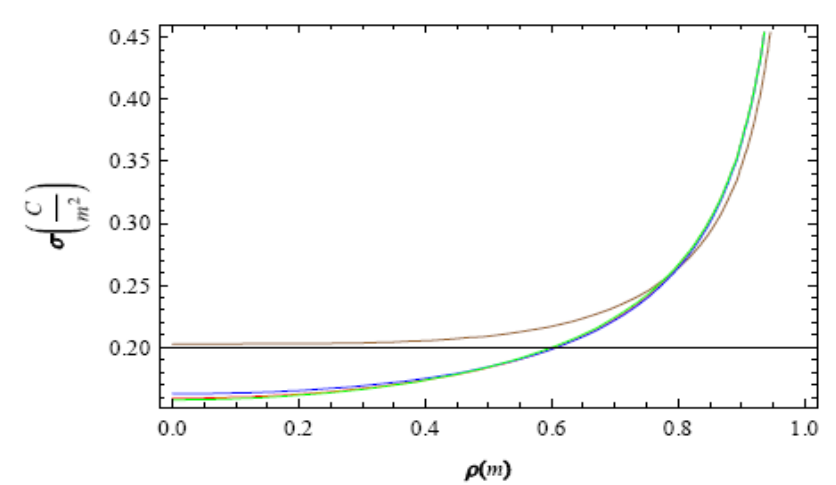

Figure 4. Surface charge distribution on the disk, based on expansion functions defined in the finite domain. Red line is for the analytic solution, brown - for one expansion function, blue - for two expansion functions, and green line is for three expansion functions

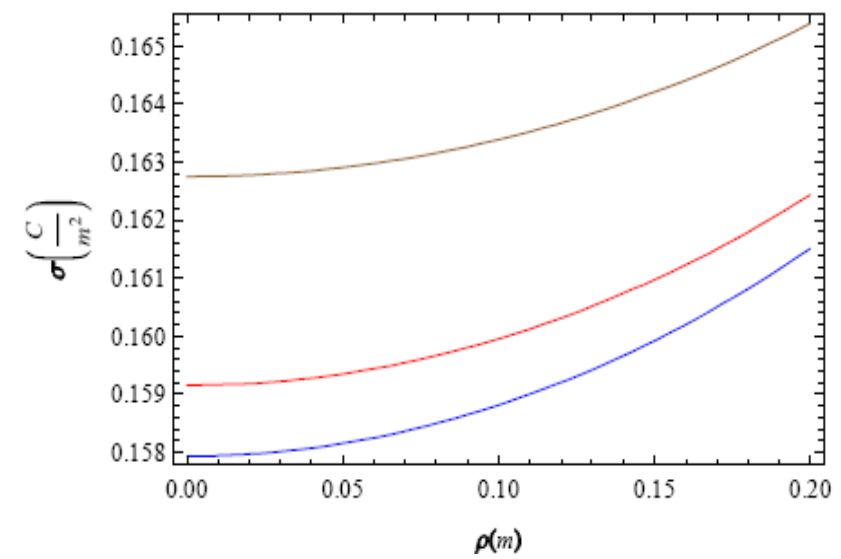

Figure 5. Surface charge distribution near the centre of the disk, based on expansion functions defined in the finite domain. Red line is for the analytic solution, brown - for two expansion functions, blue - for three expansion functions

\section{Conclusions}

The possibility to apply the moment method by using expansion functions describing the fields in the infinite domain (outside the body) has been checked, and it is shown that excellent accuracy and converging rate has been achieved, even slightly better than the case of the conventional MM solution based on expansion functions defined on the finite body. The key point was the requirement that the expansion functions will exactly obey the a-priori known physical behavior of the fields, near the edge of the body and at infinity. We conclude that efficient MM solutions based on expansion functions defined in an infinite domain is possible. The efficiency of the MM solution based on expansion functions defined in the infinite domain has to be checked in the future for electromagnetic problems, for example for antennas with different electrical sizes.

\section{Appendix}

A1. The Fourier-Bessel Transform of the 1st expansion

\section{Function Defined in the Infinite Domain}

The Fourier-Bessel transform for the MM first expansion function in the infinite domain is

$$
\begin{gathered}
\widetilde{e}_{1}(k)=\int_{R}^{\infty} e_{1}(\rho) J_{1}(k \rho) \rho d \rho \\
=\int_{R}^{\infty} \frac{J_{1}(k \rho) \rho d \rho}{\sqrt{\rho^{4}-R^{4}}}= \\
{ }_{1} F_{4}\left(\frac{1}{2} ; \frac{1}{4}, \frac{3}{4}, \frac{3}{4}, \frac{5}{4} ; \frac{k^{4} R^{4}}{256}\right)- \\
\frac{k \sqrt{\pi} R}{64}\left[\frac{32 \Gamma\left(\frac{3}{4}\right){ }_{1} F_{4}\left(\frac{3}{4} ; \frac{1}{2}, 1, \frac{5}{4}, \frac{3}{2} ; \frac{k^{4} R^{4}}{256}\right)}{\Gamma(1 / 4)}\right. \\
\left.+\frac{k^{2} R^{2} \Gamma\left(-\frac{3}{4}\right){ }_{1} F_{4}\left(\frac{5}{4} ; \frac{3}{2}, \frac{3}{2}, \frac{7}{4}, 2 ; \frac{k^{4} R^{4}}{256}\right)}{\Gamma(-1 / 4)}\right]
\end{gathered}
$$

where ${ }_{1} \mathrm{~F}_{4}$ is a generalized hypergeometric function[], and $\Gamma$ is the gamma functions[14].

\section{A2. Calculation the behaviour of the potential around the disk for $\rho \rightarrow \infty$}

A calculation for finding the behaviour of the potential at infinity is shown below[15].

$$
\begin{aligned}
& \phi(\rho, \varphi, z=0) \\
= & \frac{1}{4 \pi \varepsilon_{0}} \int_{0}^{2 \pi} d \varphi^{\prime} \int_{0}^{R} \frac{\sigma\left(\rho^{\prime}\right)}{\left|\left(x-x^{\prime}\right)^{2}+\left(y-y^{\prime}\right)^{2}\right|} \rho^{\prime} d \rho^{\prime} \\
= & \frac{1}{4 \pi \varepsilon_{0}} \int_{0}^{2 \pi} d \varphi^{\prime} \int_{0}^{R} \frac{\sigma\left(\rho^{\prime}\right) \rho^{\prime} d \rho^{\prime}}{\left|\rho^{2}+\rho^{\prime 2}-2 \rho \rho^{\prime} \cos \left(\varphi-\varphi^{\prime}\right)\right|}
\end{aligned}
$$

Due to the azimutal symmetry of the potential around the disk, we can set $\mathrm{\varphi}=0$

$$
\begin{aligned}
& 2 \pi \varepsilon_{0} \phi(\rho, \varphi, z=0) \\
& \frac{1}{2} \int_{0}^{2 \pi} d \varphi^{\prime} \int_{0}^{R} \frac{\sigma\left(\rho^{\prime}\right) \rho^{\prime} d \rho^{\prime}}{\left|\rho^{2}+\rho^{\prime 2}-2 \rho \rho^{\prime} \cos \varphi^{\prime}\right|} \\
= & \int_{0}^{R}\left\{\frac{K\left[\frac{-4 \rho \rho^{\prime}}{\left(\rho-\rho^{\prime}\right)^{2}}\right]}{\rho-\rho^{\prime}}+\frac{K\left[\frac{4 \rho \rho^{\prime}}{\left(\rho+\rho^{\prime}\right)^{2}}\right]}{\rho+\rho^{\prime}}\right\} \sigma\left(\rho^{\prime}\right) \rho^{\prime} d \rho^{\prime}
\end{aligned}
$$

where $\mathrm{K}($.$) is the Elliptic function[16]. However, we$ have the asymptotic behaviour

$$
\begin{aligned}
& \frac{2 K\left[\frac{-4 \rho \rho^{\prime}}{\left(\rho-\rho^{\prime}\right)^{2}}\right]}{\rho-\rho^{\prime}}+\frac{2 K\left[\frac{4 \rho \rho^{\prime}}{\left(\rho+\rho^{\prime}\right)^{2}}\right]}{\rho+\rho^{\prime}} \\
& \underset{\rho \rightarrow \infty}{\sim} \frac{2 \pi}{\rho}+\frac{\pi \rho^{\prime 2}}{\rho^{3}}+\frac{9 \pi \rho^{4}}{32 \rho^{5}}+\ldots
\end{aligned}
$$

Hence, we finally obtain

$$
E_{\rho} \sim \frac{\alpha}{\rho^{2}}+\frac{\beta}{\rho^{4}}+\frac{\gamma}{\rho^{6}}+\ldots, \quad \rho \rightarrow \infty
$$




\section{REFERENCES}

[1] Harrington, Roger F., Field Computation Moment Methods, IEEE Press Series on Electromagnetic Wave Theory, April 21, 1993.

[2] Rengarajan, S. R., "Choice of basis functions for accurate characterization of infinite array of microstrip reflectarray elements", IEEE Antennas and Wireless Propagation Letters, Vol. 4, 47-50, 2005.

[3] Svezhensev, A. Y., "Full wave edge accounting analysis of waves in infinite array of stub-loaded rectangular waveguides", Int. J. Infrared Milli. Vol. 29, 724-740, 2008.

[4] Matzner, H., and S. Shtrikman, "The Westmijze head - an in-depth study", Current Topics in Mag. Res,1, 145-157, 1994.

[5] Miraftab, V., R. R. Mansour and M. Yu, "Moment method using fuzzy basis functions",IEEE/MTT Int. Sym., Honolulu, 1999-2002, 2007.

[6] Matekovits, L., V. A. Laza and G. Vecchi, "Analysis of large complex structures with the synthetic-functions approach", IEEE Trans. AP, Vol. 55, No. 9, 2509-2521, 2007.

[7] Lacente, E., A. Monorchio and R. Mittra, "An iteration-free MoM approach based on excitation independent characteris- tic basis functions for solving large multisacle electromagnetic scattering problems", IEEE Trans. AP, Vol. 56, No. 4, 999-1007, 2008.

[8] Jackson, J. D., Classical Electrodynamics, John Wiley \& Sons, 89-93, 1962.

[9] Abramowitz, M., and I. A. Stegun, Handbook of Mathematical Functions, Dover Publications, Inc., 358, 1970.

[10] Jackson, J. D., Classical Electrodynamics, John Wiley \& Sons, 3rd Ed., 118, 1999.

[11] Gradshteyn, I. S., and L. M. Ryzhik, Table of Integrals, Series, and Products, Academic Press, Inc., 1045, 1980.

[12] Gradshteyn, I. S., and L. M. Ryzhik, Table of Integrals, Series, and Products, Academic Press, Inc., 1032, 1980.

[13] Mathematica Software, Wolfram Research, Champaign, IL.

[14] Abramowitz, M., and I. A. Stegun, Handbook of Mathematical Functions, Dover Publications, Inc., 255, 1970.

[15] Jackson, J. D., Classical Electrodynamics, John Wiley \& Sons, 3rd Ed., 35, 1999.

[16] Abramowitz, M., and I. A. Stegun, Handbook of Mathematical Functions, Dover Publications, Inc., 589, 1970. 